\title{
Beurteilung der Lebergröße
}

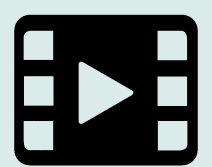

Mit Untersuchungsfilm

Es gibt zwei einfache Möglichkeiten, mit denen Sie ohne den Einsatz bildgebender Verfahren die Größe der Leber bestimmen können: Die LEBER-PERKUSSION und die sogenannte KRATZAUSKULTATION. Die Bestimmung der Lebergröße ist vor allem bei Verdacht auf Stauungsphänomene, Fettleber oder zirrhotische Veränderung des Lebergewebes angezeigt. Eine ausführliche Anamnese, Inspektion des Bauches sowie eine Leberpalpation sind zuvor vorzunehmen.

\section{Perkussion der Leber}

Wenn Sie die Lebergröße durch Perkussion bestimmen, machen Sie sich zunutze, dass luftgefüllte Bereiche eher „sonor“ klingen. Durchführung: Für die Untersuchung muss der Oberkörper des Patienten entkleidet sein. Der Patient liegt mit entspannter Bauchdecke auf der Untersuchungsliege. Dazu winkelt er die Beine an und legt die Arme neben den Körper. Hilfreich sind eine Knierolle und ein Kopfkissen.

Legen Sie für die Perkussion den Plessimeter-Finger (Mitteloder Zeigefinger) überstreckt auf den Thorax. Klopfen Sie mit der Fingerspitze des Mittel- oder Zeigefingers der anderen Hand 1-2 Mal locker aus dem Handgelenk auf das Mittelglied des Plessimeter-Fingers. Perkutieren Sie die Leber entlang einer gedachten Linie, die senkrecht durch die Mitte des Schlüsselbeins in Richtung Becken verläuft, der sogenannten Medioklavicularlinie. Eine gesunde Leber nimmt in diesem Bereich etwa 7-12 cm Raum ein. Beginnen Sie mit der Perkussion oberhalb der vermuteten oberen Lebergrenze. Perkutieren Sie nun engmaschig, also in geringen Abständen, in Richtung Becken.

Beurteilung: Das dichte Lebergewebe erkennen Sie am hyposonoren Klopfschall, dem sogenannten Schenkelschall. Dieser hebt sich nach oben gegen den sonoren Klopfschall der Lungen und nach unten gegen den normalerweise sonoren Klopfschall des Darmes ab. Die Veränderung des Klopfschalls zeigt also sowohl die obere als auch die untere Lebergrenze an.

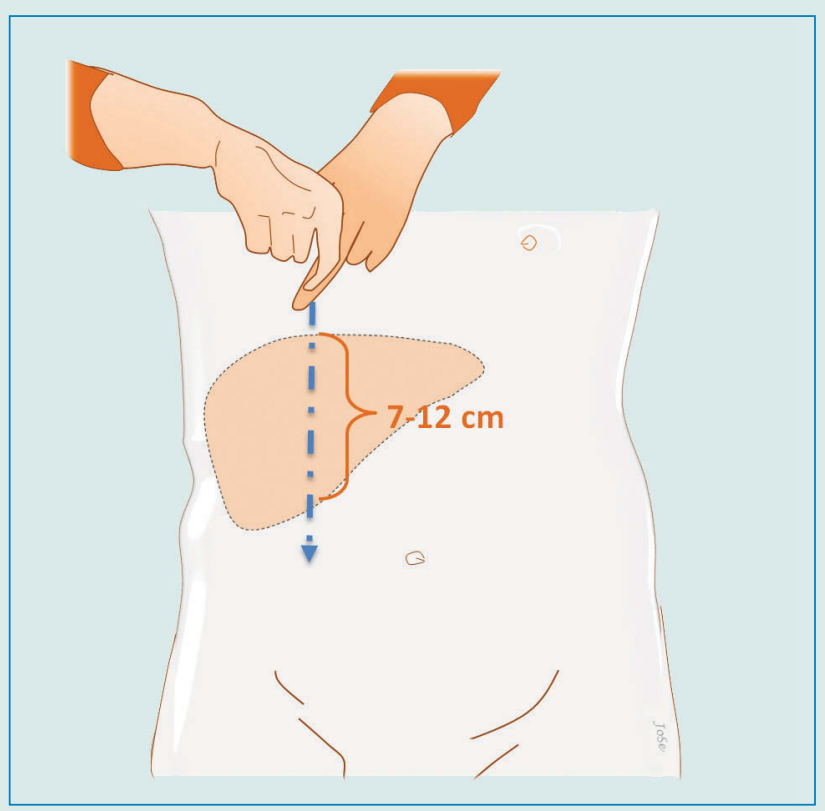

Abb.1 Man perkutiert die Leber entlang der Medioklavicularlinie.

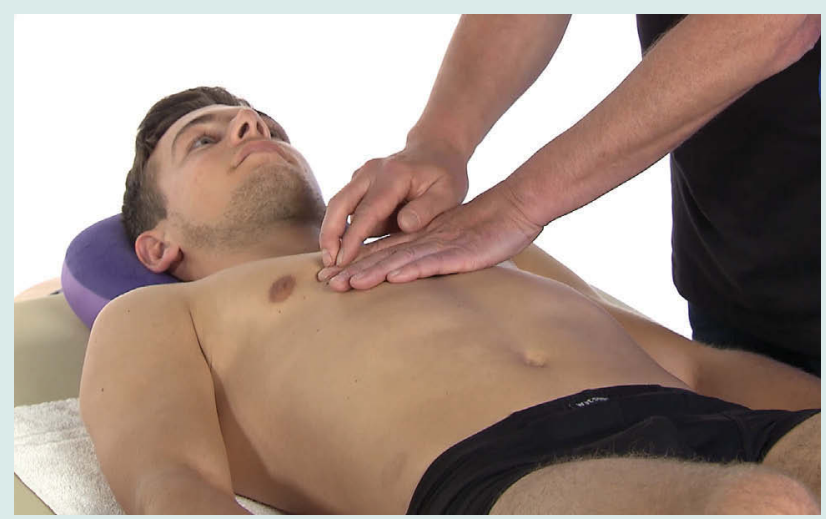

Abb.2 Perkutiert wird in geringen Abständen in Richtung Becken. 


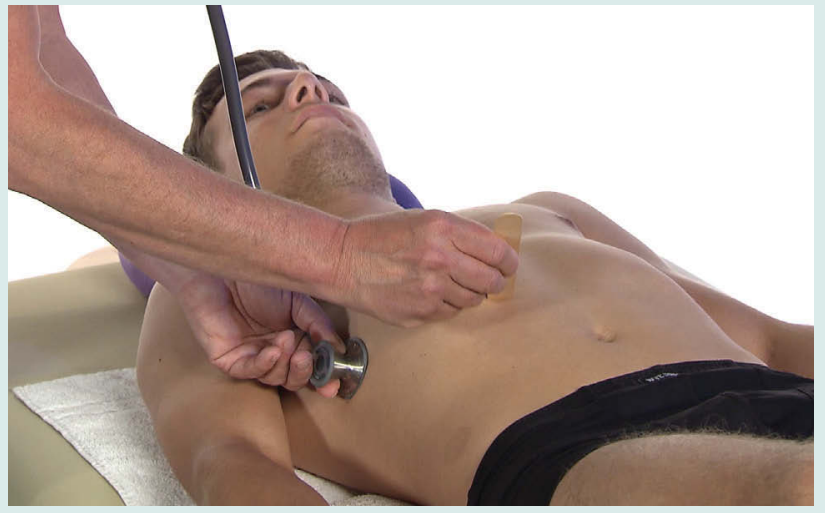

Abb.3 Bei der Kratzauskultation kratzt man mit einem Spatel in geringen Abständen kräftig entlang der Medioklavikularlinie in Richtung Becken.

\section{Kratzauskultation}

Durchführung: Für die Kratzauskultation der Leber setzen Sie das Stethoskop an der Stelle auf die Bauchwand, wo Sie Lebergewebe vermuten. Am ehesten also am unteren rechten Rippenbogen. Setzen Sie einen Holzspatel oberhalb der vermuteten oberen Lebergrenze auf. Kratzen Sie mit kleinen Bewegungen und in geringen Abständen kräftig entlang der Medioklavikularlinie in Richtung Becken.

Beurteilung: Sobald Sie mit dem Spatel über Lebergewebe sind, überträgt das Stethoskop das Kratzgeräusch. Das bedeutet: Oberhalb der Leber hören Sie das Kratzen nicht oder nur sehr leise, über der Leber hören Sie es sehr deutlich. Jenseits der unteren Lebergrenze wird das Kratzgeräusch dann allmählich leiser bis es nicht mehr zu hören ist. Das Auftauchen beziehungsweise das Verschwinden des Kratzgeräusches zeigt die Lebergrenzen an.

Grafiken: (c) Hufeland-Schule Senden/HeilpraktikerLernZentrum.de

HP Jürgen Sengebusch, HP Michael Herzog, Senden

Dieser Artikel ist online zu finden:

http://dx.doi.org/10.1055/s-0037-1599809

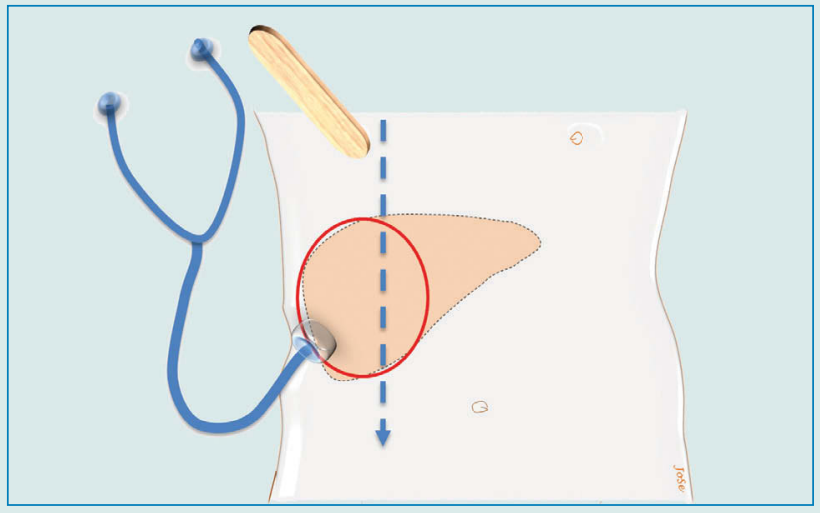

Abb. 4 Oberhalb der Leber ist das Kratzen nicht oder nur sehr leise zu hören, über der Leber sehr deutlich. Jenseits der unteren Lebergrenze wird das Kratzgeräusch allmählich leiser. 